\title{
CT and Ultrasonographic Findings in Jugular Vein Ectasia
}

\author{
Robert W. Jasinski, MD, Jonathan M. Rubin, MD†
}

Jugular vein aneurysm can mimic more serious abnormalities and produce cosmetic difficulties for patients. The existence of marked asymmetry in the sizes of normal jugular veins is known from anatomic studies. ' We present two cases in which asymmetric enlargement of the jugular vein was misinterpreted as disease in other structures. In each case, the correct diagnosis was made using ultrasound, and in one case, examination using duplex Doppler imaging documented that a pulsatile mass was venous in nature. Based on these cases, we attempted to evaluate the frequency of asymmetry in jugular vein diameters. This information should make sonographers aware of this normal variation in size between right and left jugular veins, and help them to avoid the pitfalls associated with ascribing this asymmetry to a pathologic condition.

\section{REPORT OF TWO CASES}

Casc 1. A 45-year-old man who had a history of Hodgkin's disease in complete remission for 13 years found a new nodule in the right lower neck. A computed tomographic (CT) scan with infusion (fig. 1) performed at an outside institution led to a mistaken diagnosis of confluent adenopathy in the right neck which extended along the carotid artery deep to the sternocleidomastoid. The largest area of suspected adenopatlyy was $2.5 \mathrm{~cm}$ in greatest diameter. Callium scan and physical examination results were unremarkable.

A repeat CT scan with bolus injection (fig. 2) revealed a tubular mass extending deep to the sternocleidomastoid which corresponded in anatomic location to the jugular vein, and was enhanced at the same time and to the same degree as the contralateral jugular vein. Static B-scan ultrasonic examination showed that the jugular vein on the right was more prominent than the one on the left, but there was no evidence of adenopatlyy or focal jugular vein dilation.

Case 2. A 77-year-old woman was adenitted emergently for evaluation of a suspected $4 \mathrm{~cm}$ right carotid aneurysm found at another institution using ultrasound. The patient

Received July 11, 1983, from the Department of Radiology, University of Michigan Hospitals, Ann Arbor, Michigan 48109, and the Department of Radiology. University of Chicago Hospitals and Clinics, 950 E. 59th Street, Chicago, Illinois 60637. Accepted for publication February 28, 1984

- Department of Radiology, University of Michigan Hospitals.

† Department of Radiology. University of Chicago Hospitals and Clinics.

Address correspondence and reprint requests to Dr. Rubin had occasional dysphagia but was without neurologic symptoms. Physical examination showed no evidence of carotid dilation, but fullness was recognized and a $3 \mathrm{~cm}$ pulsatile mass with a questionable bruit was described. Duplex Doppler ultrasonic (Diasonics", $7.5 \mathrm{MH}$ z) examination revealed a right internal jugular vein which was dilated focally just above the level of the clavicle. It varied appropriately in caliber with respiratory and Valsalva maneuvers (fig. 3) and produced the pulsed Doppler waveform of a venous structure (fig. $4 A$ ), with velocity opposite that of the carotid artery (fig. $4 B$ ).

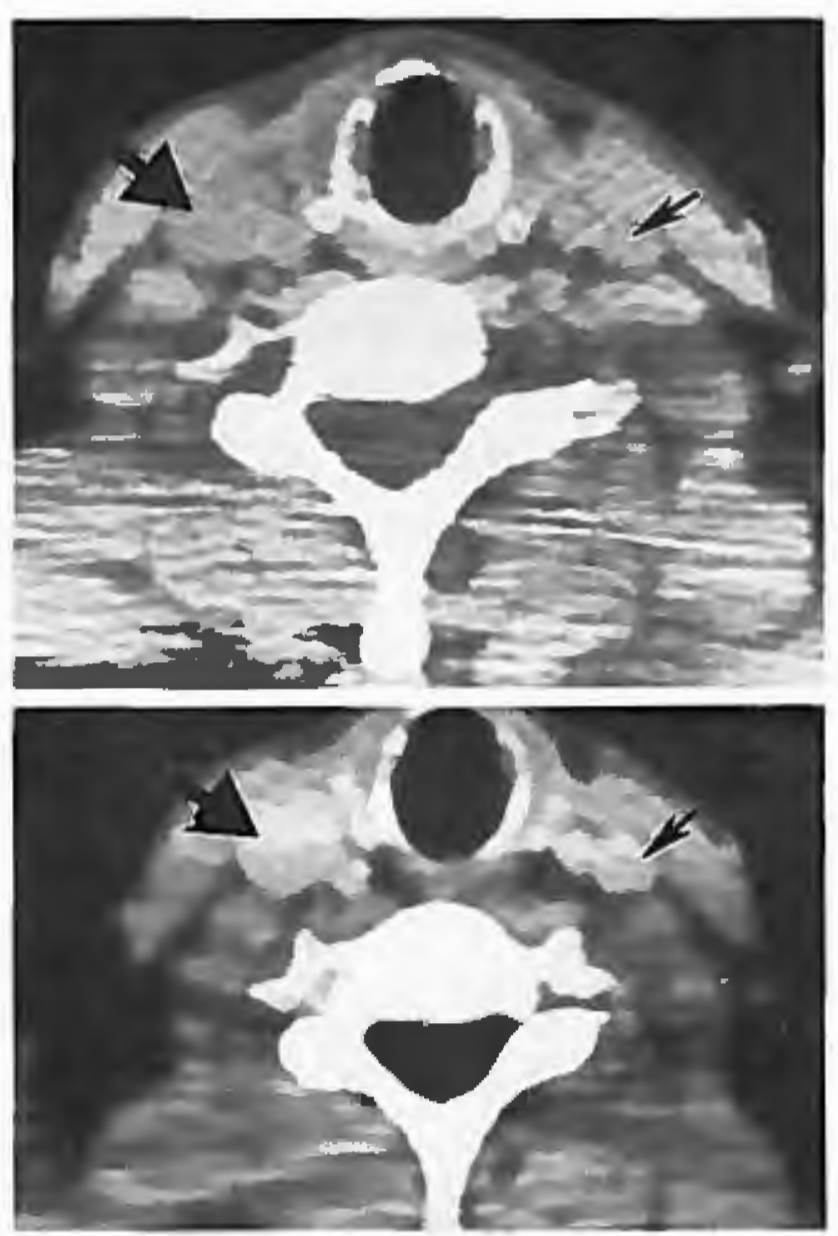

Figure 1 (top). Right internal jugular vein (large arrow) thought to represent adenopathy on initial examination, and left internal jugular vein (small arrow).

Figure 2 (bottom). Subsequent examination using contrast bolus technique enhances the great vessels of the neck and reveals their true vascular identity. 

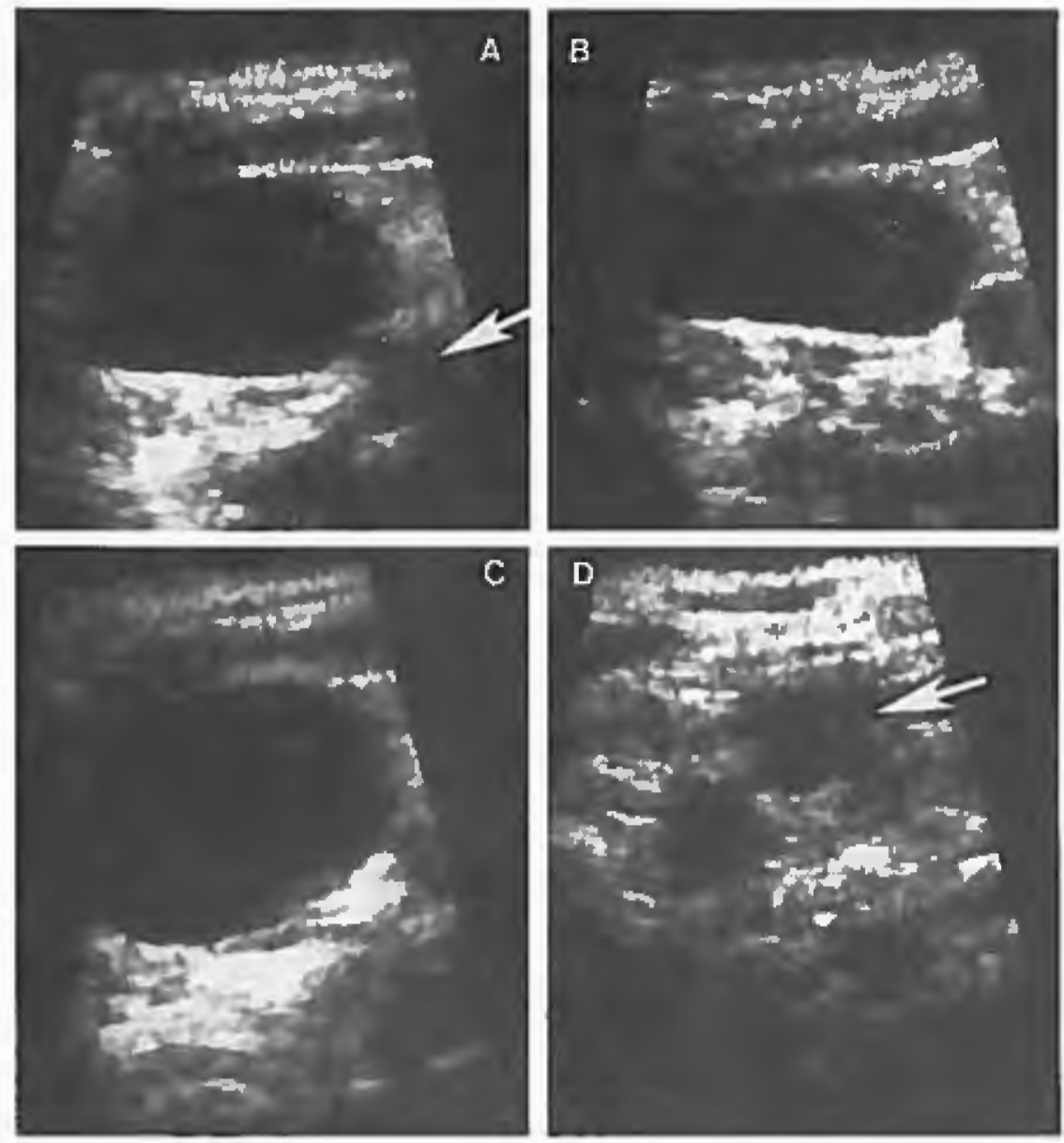

Figure 3. Dilated right internal jugular wein anterolat* eral to carotid artery (white arrow) during expiration ( $A, 3$ $\times 1,5 \mathrm{~cm}$ ), during inspiration $(B, 3 \times 1,1 \mathrm{~cm})$, and during the Val sa va maneuver $(C, 3$ $\times 2 \mathrm{~cm}$ ). $D=$ contralateral normal left jugular vein for comparison $(1 \times 0.8 \mathrm{~cm})$ (arrow).

\section{MATERIALS AND METHODS}

In an attempt to cletermine the variability in size between normal right and left jugular veins, routine cliest CT scans (Siemens Somotome II ${ }^{\%}$ ) of 34 patients without mediastinal masses or histories of mediastinal disense processes were reviewed. The largest calibers of the right and left internal jugular veins were measured $1 \mathrm{~cm}$ above the level of the pulmonary apices. Scans were performed during inspiration.

\section{RESULTS}

The mean diameters of the right and left internal jugular veins were $1.5 \mathrm{~cm}$ (SD $=0.4 \mathrm{~cm}$ ) and 1.3 cm (SD $=0.4 \mathrm{~cm}$ ) respectively with six calses in which the left jugular vein was larger (mean difference $=0.4 \mathrm{~cm}, \mathrm{SD}=0.3 \mathrm{~cm}$, eight cases in which there was no difference, and 20 cases in which the right diameter was greater than the left (mean difference $=0.6 \mathrm{~cm}, \mathrm{SD}=0.3 \mathrm{~cm})$.

\section{DISCUSSION}

Jugular vein aneurysm presents as a neck mass which increnses with size on straining. coughing, crying, and other respiratory maneuvers, ${ }^{n .1}$ It is usually asymptomatic, may enlarge over time and, in the adult, may date to childhood. Symptoms that have been reported in association with jugular vein ectasia include shoulder pain, weakness of the ipsilateral hand, hoarseness, pain and lower respiratory tract symptoms. ${ }^{2,4}-7$ The ectasia may involve the internal and external jugular veins on either side of the neck, and it is said to occur more often on the left in adults. ${ }^{8}$ Such a left-sided distribution is not supported for the internal jugular vein, at least on the basis of several reports, ${ }_{2}, 3,5,6,8.9$ in which the tendency was for right internal jugular vein dilation.

Causes of aneurysmal jugular vein include trauma, inflammation, arteriovenous fistula, congenital weakness or degenerative changes in the wall of the vein, ${ }^{9}$ cervical rib, Valsalva type maneuvers, ${ }^{4}$ or pectus excavatum. ${ }^{5}$ In venous aneurysins, vein wall composition has been reported to be both normal ${ }^{3,5, y}$ and abnormal, ${ }^{5,6}$ which may reflect the heterogeneity of possible causes. In those cases of abnormal wall composition, it is the elastic and muscular layers that are defective , $^{5,6,10}$

In the differential diagnoses of neck masses, both real and pseudo-lesions should be considered. Pseudo-lesions include distention of the jugular 
Figure 4. A (lop), pulsed Doppler examination of the right common carotid artery with waveform showing flow away from the heart as a positive velocity (white arrow). B (bottom), pulsed Doppler examination of the dilated right jugular vein with sample volume proximally located (small arrow). Velocity during inspiration (large arrow) is markedly negative, as expected, in contradistinction to the carotid waveform.
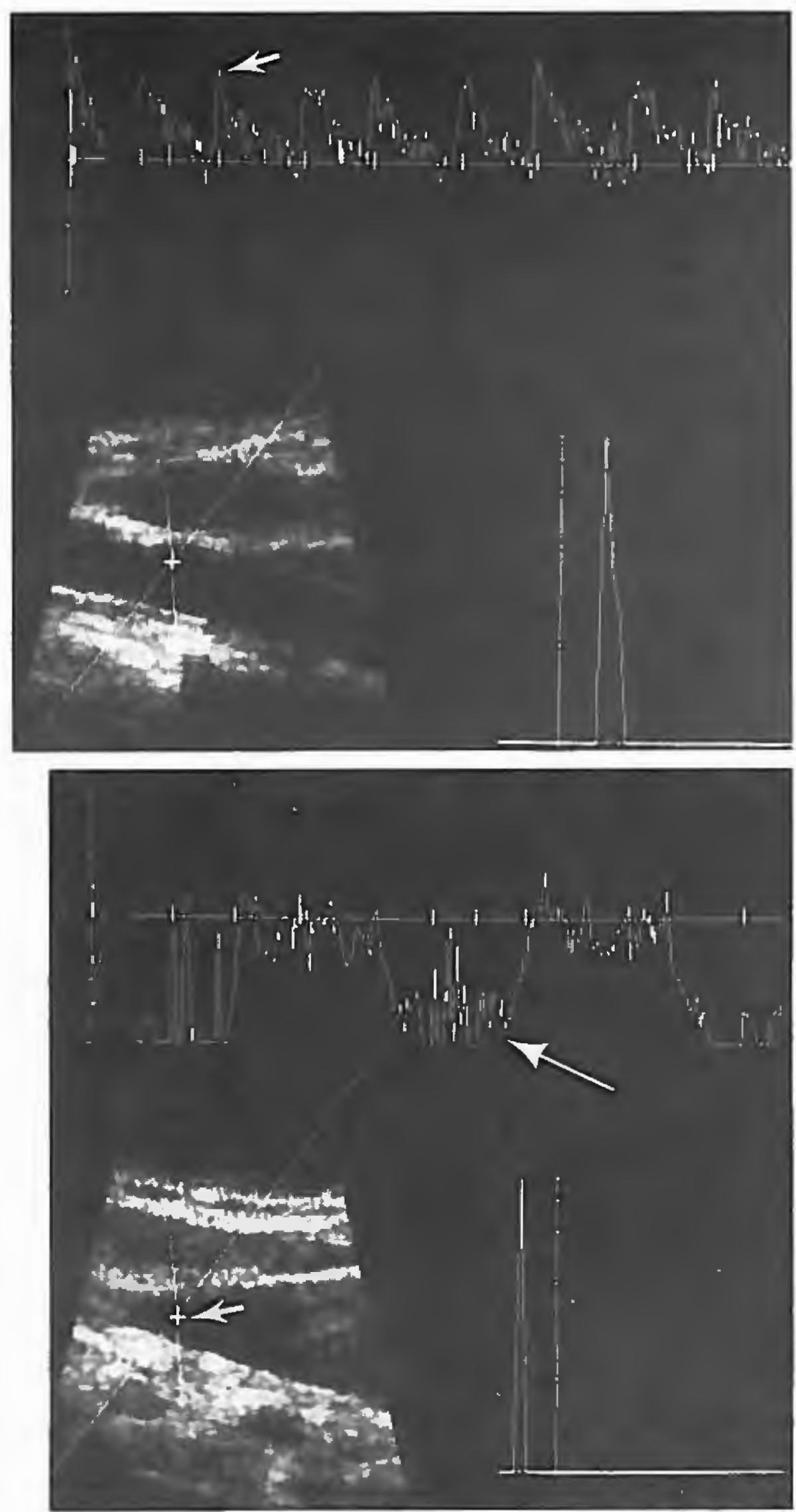
vein related to arm positioning and Valsalva maneuver ${ }^{11}$ and the scalenus anticus syndrome. ${ }^{7}$ In normal persons, the right internal jugular vein is usually larger than the left. This has been attributed to the tendency of the straight sinus to empty more frequently to the right transverse sinus rather than to the left transverse sinus. Therefore, proportionately more blood is delivered from the cranial venous system to the right jugular vein. ${ }^{1}$ If pronounced, this tendency would produce relative dilation of the right jugular vein; but, unlike jugular vein aneurysm, there is no focal dilation with this condition. Because focal dilation was not present, case 1 probably represented a variation of the normal asymmetry in jugular vein size. Although pathologic proof is lacking, case 2 probably was not a variation of normal, but rather a true aneurysm, because of the focal ectasia seen on ultrasound and the focal palpable fullness.

Other true lesions that produce neck masses include cavernous hemangiomas, branchial and enterogenous cysts, lymphoceles, laryngeal diverticula, cervical adenitis, and persistent jugular Jymph sac. ${ }^{7}$ Findings other than jugular vein ectasia that increase in size with Valsalva maneuver include tumors or cysts of the superior mediastinum, external laryngeal diverticula, and persistent jugular lymph sac, ${ }^{-12}$ The tubular nature of the dilated jugular vein distinguishes it from nonvascular fluid collections; its velocity opposite that of the carotid, and changes in this velocity and in vessel caliber witl respiratory maneuvers allow it to be distinguished from an arterial abnormality.

Computed tomograplice scanning with infusion can reliably demonstrate the vascular nature of jugular vein ectasia, and therefore allow it to be distinguished from nonvascular lesions such as lymphadenopathy. Duplex Doppler real-time ultrasonic imaging can demonstrate appropriate changes in size and flow with respiratory maneuvers, thus differentiating solid or cystic masses from vascular structures. Unlike CT scanning, pulsed Doppler imaging does not require administration of contrast medium, irradiation, or great expense to characterize vascular neck lesions.

If available, duplex Doppler ultrasound should be the initial choice in the investigation of suspected vascular neck masses. The sonographer should be aware of the variations in normal jugular vein sizes to avoid overdiagnosis of neck lesions.

\section{REFERENCES}

1. Romanes GJ. Cunningham's Manual of Practical Anatomy. Volume 3, ed. 13. London. Oxlord Universlty Press. 1966, p 131

2. LaMonte SJ, Walker EA. Moran WB: Internal jugu'ar phlebectasia. Arch Otolaryngol 102.706, 1976

3. Stevens RK, Fried AM, Hood TR: Ulitrasonic diagnosis of jugu'ar venous aneurysm. J Clin Ultrasound 10:85 1982

4. Rowe MJ: Contracture of the scalenus anterior causing aneurysmal varix of right internal jugular ve.n. J Bone Joint Surg 28:147, 1946

5. Okay NH, Bryk D, Kroop IG, el al: Phlebectasia of the jugular and great mediastinal veins. Radiology $95.629,1970$

6. Pataro VF, Crosbie JC, Conde RM Jugular phlebectasias. J Cardiovasc Surg (Torino) 2.3.1961

7. Geary FJ. Altman AR, Borrelli FJ, et al: Pectus excavatum as cause of compressed innominate vein syndrome. NY State J Med 66 1346, 1966

8. Gordon DH, Rose JS, Kottmeier P. et al: Jugular venous ectasia in children Radiology 118 147, 1976

9. Gilbert MG, Greenberg LA, Brown WT, et al. Fusiform venous aneurysm of the neck in children. J Pediatr Surg 7:106, 1972

10. Schatz IJ, Fine $G$ Venous aneurysms. N Engl J Med 266:1310. 1962

11. Yeh $E$, Pohmenn GP. Ruelz PP, et al Jugular venous reflux in cerebral radionucl.de angiography. Rad ology 118:730. 1976

12. Ste-inberg I, Watson RC: Lymphangiographic and angiographic diagnosis of pers stent jugu ar iymph sac. N Engl J Med 2751471,1966 